Pacific Journal of Mathematics

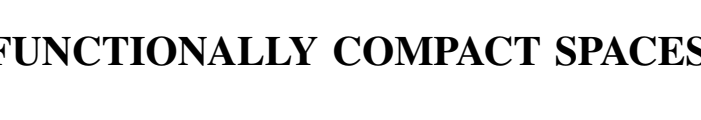




\title{
FUNCTIONALLY COMPACT SPACES
}

\author{
R. F. Dickman, JR., AND A. Zame
}

The purpose of this note is to introduce a property which is weaker than compactness but stronger than minimality which closely relates to some filter properties and some mapping properties of compact spaces. We also indicate some procedures for constructing absolutely closed and minimal spaces.

All of the definitions used but not given in this paper may be found in [2].

Definition. An open filter base on a topological space $X$ is a filter base consisting of open subsets of $X$.

A Hausdorff topological space $X$ is called functionally compact if whenever $\mathscr{U}$ is an open filter base on $X$ such that the intersection $A$ of the elements of $\mathscr{W}$ is equal to the intersection of the closures of the elements of $\mathscr{U}$, then $\mathscr{U}$ is a base for the neighborhoods of $A$.

THEOREM 1. There exists a noncompact Hausdorff space which is functionally compact.

Proof. Let $Z^{+}=$positive integers, $I=[0,1]$. Let $0<a_{1}<a_{2}<\cdots$ $<a_{n}<\cdots$ be an increasing sequence in $I$ with $\lim a_{n}=a_{0}$. For each $i \in Z^{+}$let $a_{i}^{i}$ be a strictly increasing sequence (in $\left(a_{i-1}, a_{i}\right)$ for $i \geqq 2$; in $\left(0, a_{1}\right)$ for $\left.i=1\right)$ with $\lim _{j} a_{i}^{j}=a_{i}$. Let

$$
C=\bigcup_{i>0}\left\{a_{i}\right\} \cup \bigcup_{\substack{i>1 \\ j>1}}\left\{a_{i}^{j}\right\}
$$

Let $\alpha_{0}=a_{0}$ and for $i \geqq 1$ let

$$
\alpha_{i}=\left\{a_{1}^{i}, a_{2}^{i-1}, \cdots, a_{i}^{1}, a_{i}\right\} .
$$

Let $C^{*}=\left\{\alpha_{i}: i \geqq 0\right\}$ and put

$$
F=(I \backslash C) \cup C^{*} .
$$

Let $\mathscr{T}$ be the topology on $F$ such that $\mathscr{T} \mid(I \backslash C)$ is the usual topology on $I \backslash C$ and a basic open neighborhood of $\alpha_{n}(n \geqq 0)$ is a set of the form $N \cup\left\{\alpha_{n}\right\}$, where $N=O \cap(I \backslash C), O$ open in $I$ and $\left\{a_{1}^{n}, \cdots, a_{n}^{1}\right.$, $\left.a_{n}\right\} \subset O$.

It is easy to see that $(F, \mathscr{T})$ is a Hausdorff space. It is not compact, however, because $C^{*}$ is an infinite discrete closed set in $F$. 
In fact, if $O_{1}, O_{2} \in \mathscr{T}$ and $O_{1} \cap C^{*} \neq \varnothing, O_{2} \cap C^{*} \neq \varnothing$ then $\operatorname{cl}_{F} O_{1} \cap$ $\mathrm{cl}_{F} \mathrm{O}_{2} \cap C^{*}$ is infinite, so that points in $C^{*}$ cannot be separated by closed neighborhoods. We will now show that $(F, \mathscr{T})$ is functionally compact.

Define the set function $\psi: F \rightarrow I$ via

$$
\psi(\{a\})=\left\{\begin{array}{l}
a \text { if } a \in I \backslash C \\
a_{0} \text { if } a=\alpha_{0} \\
\left\{a_{1}^{i}, \cdots, a_{i}^{1}, a_{i}\right\} \text { if } a=\alpha_{i}, i \geqq 1
\end{array}\right.
$$

and $\psi(A)=\bigcup_{a \in A} \psi(\{a\})$ for $A \subseteq F . \quad \psi$ does not preserve openness or closedness. However, if $A \subseteq F$ is closed then there exists a set $A^{c}$, closed in $I$, such that

and

( i ) $\psi(A) \cap(I \backslash C)=A^{c} \cap(I \backslash C)$

(ii) $\psi(A) \supseteqq A^{c}$,

namely,

$$
A^{c}=c l_{I} \psi(A) \cap(I \backslash C) .
$$

Hence, if $U \subseteq F$ is open there exists an open set $U^{0} \cong I$ such that

(iii) $U^{0} \cap(I \backslash C)=\psi(U) \cap(I \backslash C)$

(iv) $U^{0} \supseteqq \psi(U)$;

namely, the set $U^{0}=\left(\left(U^{\prime}\right)^{c}\right)^{\prime}$ (where ' denotes complementation in either $F$ or $I$ ).

Now, suppose $\mathscr{U}$ is an open filter in $F$ such that $\cap\{U: U \in \mathscr{U}\}=$ $\cap\left\{c l_{F} U: U \in \mathscr{U}\right\}=A$ and that $V \supseteqq A, V \in \mathscr{T}$. We claim that there exist $U_{1}, U_{2} \in \mathscr{T}$ such that $V \supseteqq U_{1} \cap U_{2}$.

First of all, if $O \in \mathscr{T}$ and $O \cap C^{*}$ is infinite, then $c l_{F} O \supseteqq C^{*}$. Hence if $A \cap C^{*}$ is finite then there exists $U \in \mathscr{U}$ such that $U \cap C^{*}=$ $A \cap C^{*}$, while if $A \cap C^{*}$ is infinite then $A \supseteqq C^{*}$. Thus, in either case there exists $U_{1} \in \mathscr{U}$ such that $V \cap C^{*} \supseteqq U_{1} \cap C^{*}$. Furthermore,

$$
V^{0} \supseteqq \psi(A)=\cap \psi\left(c l_{F} U\right) \supseteqq \cap\left(c l_{F} U\right)^{c} \text {. }
$$

Hence, since $V^{0}$ is open in $I$ and $\left(c l_{F} U\right)^{c}$ is closed in $I$ the compactness of $I$ implies that there exists $U_{2} \in \mathscr{U}$ such that $V^{0} \supseteq\left(c l_{F} U_{2}\right)^{c}$. But then

$$
\begin{aligned}
V^{0} \cap(I \backslash C) & =\psi(V) \cap(I \backslash C)=V \cap(I \backslash C) \supseteqq\left(c l_{F} U_{2}\right)^{c} \cap(I \backslash C) \\
& =\left(c l_{F} U_{2}\right) \cap(I \backslash C) \supseteqq U_{2} \cap(I \backslash C) .
\end{aligned}
$$

Hence

$$
V=(V \cap(I \backslash C)) \cup\left(V \cap C^{*}\right) \supseteqq\left(U_{2} \cap(I \backslash C)\right) \cup\left(U_{1} \cap C^{*}\right) \supseteqq U_{1} \cap U_{2} .
$$

This proves the result. 
There are some obvious generalizations of this construction. The above example $F$ of a functionally compact space also shows that the property of being functionally compact is not closed-hereditary or openhereditary or even regular-closed-hereditary.

THeorem 2. A Hausdorff space $X$ is minimal Hausdorff if and only if for every point $x \in X$ and every open filter-base $\mathscr{C}$ on $X$ such that $x=\cap\{U: U \in \mathscr{U}\}$ and $x=\cap\left\{c l_{x} U: U \in \mathscr{C}\right\}$, $\mathscr{C}$ is a base for the neighborhoods of $x$.

Proof of the necessity. Suppose that $X$ is minimal Hausdorff and that $\mathscr{C}$ is an open filter-base on $X$ and $x \in X$ such that

$$
x=\cap\{U: U \in \mathscr{Q}\} \text { and } x=\cap\left\{c l_{x} U: U \in \mathscr{L}\right\}
$$

and let $R$ be any open set containing $x$. We note that $x$ is the unique cluster point of $\mathscr{C}$. Thus by Theorem 1.3 of [1], $\mathscr{C}$ converges to $x$ and so there exists $U \in \mathscr{U}$ such that $\mathscr{Z} \subset R$. Of course this implies that $\mathscr{C}$ is a base for the neighborhoods of $x$.

Proof of the sufficiency. We need to show that every open filterbase with a unique cluster point converges to that point. To this end let $\mathscr{Y}$ be an open filter-base on $X$ with a unique cluster point $x$ and let $R$ be any open subset of $X$ containing $x$. Let $y /$ be the collection of all open subsets of $X$ containing $x$ and let

$$
\mathscr{Q}=\{V \cap W: V \in \mathscr{Y} \text { and } W \in \mathscr{Y}\} \text {. }
$$

Then $\mathscr{C}$ is an open filter-base on $X$,

$$
x=\cap\{U: U \in \mathscr{C}\} \text { and } x=\cap\left\{c l_{x} U: U \in \mathscr{C}\right\} \text {. }
$$

Thus by our hypothesis there exists $U \in \mathscr{C}$ such that $U \subset R$. Of course this implies that $\mathscr{F}$ converges to $x$ and this completes the proof.

COROLLARY 2.1. Every functionally compact Hausdorff space is minimal Hausdorff.

Proof. This result is an immediate consequence of the definition of functionally compact and Theorem 2 .

The example due to Urysohn in Remark 1.5 of [1] is a minimal Hausdorff space which is not functionally compact. In the next section we indicate a method of constructing many such spaces.

Corollary 2.2. A functionally compact Hausdorff space is com- 
pact if and only if it is regular.

Proof. The proof follows immediately from the fact that a minimal Hausdorff is compact if and only if it is regular.

Definitions. By a mapping we will always mean a continuous function. A mapping from a space $X$ to a space $Y$ is closed provided for every closed set $C$ in $X, f(C)$ is a closed set in $Y$.

THEOREM 3. A Hausdorff space $X$ is functionally compact if and only if every mapping of $X$ into any Hausdorff space is closed.

Proof of the necessity. Suppose that $X$ is functionally compact and let $f$ be a mapping of $X$ into a Hausdorff space $Y$. Let $C$ be a closed set in $X$ and suppose there exists a point $y$ in $c l_{Y} f(C) \backslash f(C)$. Let $\mathscr{V}$ be the collection of all open subsets of $Y$ containing $y$ and let $\mathscr{C}=\left\{f^{-1}(V): V \in \mathscr{V}\right\}$. By Theorem $2, X$ is minimal Hausdorff and hence absolutely closed. Since continuous images of absolutely closed spaces are absolutely closed, $f(X)=c l_{Y} f(X)$ and so $y \in f(X)$. Thus $\mathscr{U}$ is a collection of nonempty open subsets of $X$ and therefore is an open filter-base on $X$. Furthermore since $Y$ is a Hausdorff space, $f^{-1}(y)=\cap\{U: U \in \mathscr{C}\}$ and $f^{-1}(y)=\cap\left\{c l_{X} U: U \in \mathscr{Q}\right\}$. Thus by our hypothesis $\mathscr{U}$ is a base for the neighborhoods of $f^{-1}(y)$ and so there exists $U \in \mathscr{Z}$ such that $U \subseteq X \backslash C$. But then $f(U)$ is an open subset of $f(X)$ that contains $y$ and misses $f(C)$ (since $U=f^{-1}(f(U))$ if $U \in \mathbb{C}$ ) and this is a contradiction. Thus $f(C)$ is closed and this completes the proof of the necessity.

Proof of the sufficiency. Suppose that every mapping of $X$ into a Hausdorff space is closed and let $\mathscr{U}$ be an open filter-base on $X$ such that the intersection $A$ of the elements of $\mathscr{U}$ equals the intersection of the closures of the elements of $\mathscr{U}$. Suppose further that there exists an open set $R$ of $X$ containing $A$ such that for every $U \in \mathscr{U},(X \backslash R) \cap U \neq \Phi$. Let $Y$ be the decomposition of $X$ whose only nondegenerate element is $A$ and let $f$ be the natural transformation of $X$ onto $Y$ defined by $x \in f(x)$. We topologize $Y$ by defining a base $\mathscr{B}$ for a topology as follows:

$B \in \mathscr{B}$ if and only if (i) $f^{-1}(B)$ is an open subset of $X \backslash A$; or

(ii) $f^{-1}(B) \in \mathscr{U}$.

Then $Y$ with this topology is a Hausdorff space and $f$ is a mapping of $X$ onto $Y$. By our hypothesis $f$ must be a closed map; however 
$f(X \backslash R)$ is not closed since $f(A)$ is a limit point of $f(X \backslash R)$ and $f(A) \notin f(X \backslash R)$. This is a contradiction and this completes the proof.

REMARK. The space $F$ of Theorem 1 and Theorem 3 show that the property of being functionally compact is not productive. In fact, even if $\mathscr{C}$ is compact $F \times \mathscr{C}$ will not usually be functionally compact since the projection $\pi: F \times \mathscr{C} \rightarrow \mathscr{C}$ is not a closed map unless $\mathscr{Y}$ is finite.

CoROLlary 3.1. Let $X$ be a Hausdorff space, $Z$ a functionally compact Hausdorff space and $h$ a mapping of $Z$ onto $X$. Then $X$ is functionally compact.

Proof. Let $f$ be a mapping of $X$ into a Hausdorff space $Y$ and let $C$ be a closed subset of $X$. Since $Z$ is functionally compact, the mapping $f \circ h$ is a closed map of $Z$ into $Y$ and so $f(C)=(f \circ h)\left(f^{-1}(C)\right)$ is a closed subset of $Y$. Thus $f$ is closed and $X$ is functionally compact.

CoRollary 3.2. If a Hausdorff space $X$ is the union of finitely many functionally compact spaces $X_{1}, X_{2}, \cdots, X_{n}, X$ is functionally compact.

Proof. Let $f$ be a mapping of $X$ into a Hausdorff space $Y$ and let $C$ be a closed subset of $X$. Then each of the restricted mappings $f \mid X_{i}, i=1,2, \cdots, n$, is closed and so $f(C)=\cup\left\{\left(f \mid X_{i}\right)\left(C \cap X_{i}\right): i=\right.$ $1,2, \cdots, n\}$ is closed in $Y$. Thus $f$ is closed and $X$ is functionally compact.

Definition. A closed subset $C$ of a space $X$ is said to be $r$-closed if whenever $B$ is closed in $C, x \notin B$ there exist disjoint open sets in $X$ containing $x$ and $B$, respectively.

THEOREM 4. An r-closed subset $C$ of a functionally compact space $X$ is functionally compact.

Proof. Let $\mathscr{Q}$ be an open filter-base on $C$ such that

$$
\cap\{U: U \in \mathscr{U}\}=\left\{c l_{c} U: U \in \mathscr{U}\right\}=A .
$$

Let $\mathscr{V}$ be the open filter-base on $X$ consisting of all open sets $V$ of $X$ such that $V \cap C \in \mathscr{U}$.

Then since $C$ is an $r$-closed subset of $X, \cap\{V: V \in \mathscr{Y}\}=A=$ $\left\{c l_{x} V: V \in \mathscr{V}\right\}$ and since $X$ is functionally compact, $\mathscr{V}$ is a base for 
the neighborhoods of $A$. Of course this implies that $\mathscr{U}$ is a base for the neighborhoods of $A$ relative to $C$. Hence $C$ is functionally compact.

It is also easy to see that if $C$ is an open and closed subset of a functionally compact space $X$ then $C$ is functionally compact.

2. Some related examples. Urysohn has given an example of an nonminimal absolutely closed space (Example 1.4 of [3]) and an example of a noncompact minimal space (Remark 1.5 of [1]). We will give here two general methods for constructing such spaces. In fact, our minimal spaces will be nonfunctionally compact, as is Urysohn's.

EXAMPLE 1. Let $(X, \mathscr{K})$ be a compact Hausdorff space such that $Y \subseteq X$ is an infinite closed subset, int ${ }_{\mathscr{C}} Y=\varnothing$. Let $\mathscr{J}$ be any topology on $Y$ strictly stronger than $\mathscr{K} \mid Y$, e.g., the discrete topology, and let $\mathscr{C}$ be the following topology on $X: \mathscr{C}|(X \backslash Y)=\mathscr{K}|(X \backslash Y)$ and a basic open set intersecting $Y$ is of the form $(\mathcal{O} \cap(X \backslash Y)) \cup T$, where $T \subseteq \mathscr{O} \cap Y, \mathscr{O} \in \mathscr{K}, T \in \mathscr{T}$. Then $(X, \mathscr{C})$ is a nonminimal Hausdorff space (since $\mathscr{C}$ is stronger than $\mathscr{K}$ ) and $\operatorname{int}_{\mathscr{Z}} Y=\varnothing$. However, we claim that $(X, \mathscr{C})$ is absolutely closed.

Let $\mathscr{C}=\left\{C_{\alpha}: \alpha \in \mathscr{A}\right\}$ be a $\mathscr{C}$-open covering of $X$. Notice that for each $U \in \mathscr{Q}$ there exists $K \in \mathscr{K}$ such that $K \supseteqq U$ and $K \cap(X \backslash Y)=$ $U \cap(X \backslash Y)$. Thus, if $K_{\alpha}$ is such a set for $C_{\alpha}$ then $\left\{K_{\alpha}: \alpha \in \mathscr{A}\right\}$ is a $\mathscr{K}$-open covering of $X$. Hence there exist $\alpha_{1} \cdots, \alpha_{n}$ such that

$$
K_{\alpha_{1}} \cup \cdots \cup K_{\alpha_{n}} \supseteqq X
$$

so that

$$
C_{\alpha_{1}} \cup \cdots \cup C_{\alpha_{n}} \supseteqq X \backslash Y
$$

and hence

$$
\operatorname{cl}_{\varkappa} C_{\alpha_{1}} \cup \cdots \cup c l_{\varkappa \iota} c_{\alpha_{n}}=X .
$$

This is a generalization of one of Urysohn's examples (Example 1.4 of [3]): Let $a_{i}^{j} \rightarrow a_{i}$ be disjoint convergent sequences and let $X=$ $\bigcup_{i, j}\left\{a_{i}^{j}\right\} \cup \bigcup_{i}^{j}\left\{a_{i}\right\} \cup\{\infty\}$ be the 1-point compactification of the union of these sequences and their limit points. Let $Y=\left\{a_{1}, a_{2}, \cdots\right\} \cup\{\infty\}$ and let $\mathscr{T}$ be the discrete topology on $Y$. Then $(X, \mathscr{C})$ is Urysohn's example.

EXAMPle 2. Let $(X, \mathscr{K})$ be a compact Hausdorff space, $Y \cong X$ and $\varphi$ a function from $Y$ into the 2-element subsets of $Y$ such that

(i) $Y$ is closed; 
(ii) $\operatorname{int}_{\mathscr{X}} Y=\varnothing$

(iii) there exist $x_{1}, x_{2} \in Y, x_{1} \neq x_{2}$ such that $x_{i} \in c l_{\mathscr{T}^{-}}\left(Y \backslash\left\{x_{1}, x_{2}\right\}\right)$ $(i=1,2)$;

( $\alpha) \varphi\left(z_{1}\right) \cap \varphi\left(z_{2}\right)=\varnothing$ if $z_{1} \neq z_{2}$;

(B) $\mathbf{U}_{y \in Y} \varphi(y)=Y$;

(y) there exist $y_{1} \neq y_{2}$ such that $x_{i} \in \varphi\left(y_{i}\right), i=1,2$;

$(\delta)$ for each $\mathscr{O}_{1}, \mathscr{O}_{2} \in \mathscr{K}$ such that $x_{i} \in \mathcal{O}_{i}, i=1,2$ there exists $y \in Y$ such that $\varphi(y) \cap \mathscr{O}_{i} \neq \varnothing, i=1,2$.

[For instance, we could take $X=[0,1], Y=\left\{a_{i}^{j}: i=1, \cdots, 4, j \geqq 1\right\} \cup$ $\left\{a_{1}, a_{2}, a_{3}, a_{4}\right\}$, where the $a_{i}$ are distinct and the $\left(a_{i}^{j}\right)$ are disjoint sequences of distinct points with $\lim _{j} a_{i}^{j}=a_{i}$. We can let $\varphi$ be a function which "identifies" $a_{1}^{j}$ and $a_{2}^{j}, a_{3}^{j}$ and $a_{4}^{j}, a_{1}$ and $a_{3}, a_{2}$ and $a_{4^{\circ}}$.]

Let $Y^{*}=\{\varphi(y): y \in Y\}$ and denote $\varphi(y)$ by $y^{*}$. Let $X^{*}=$ $(X \backslash Y) \cup Y^{*}$. Let $\mathscr{T}$ be the topology on $X^{*}$ defined as follows: $U \in \mathscr{T} \Leftrightarrow$ there exists $\mathscr{O} \in \mathscr{K}$ such that

(1) $U \cap(X \backslash Y)=\varnothing \cap(X \backslash Y)$

(2) for each $y^{*} \in U \cap Y^{*}$ there exists $V \in \mathscr{K}, \varphi(y) \subseteq V, V \subseteq \mathcal{O}$, such that if $\varphi(z) \subseteq V$ then $z^{*} \in U$.

It is to check that $\mathscr{T}$ is actually a topology and that $\mathscr{T}$ is Hausdorff. However, if $\mathcal{O}_{1}, \mathcal{O}_{2} \in \mathscr{T}, y_{1}^{*} \in \mathcal{O}_{1}, y_{2}^{*} \in \mathcal{O}_{2}$ then $(\delta)$ implies that $c l_{3} \mathscr{O}_{1} \cap c l_{3} \mathscr{O}_{2} \neq \varnothing$. In particular, $\mathscr{T}$ is not compact.

Notice that if $C \subseteq X^{*}$ is closed then there exists a closed set $C^{c} \subseteq X$ such that

$$
C^{c} \cap(X \backslash Y)=c \cap\left(X^{*} \backslash Y^{*}\right)
$$

and

$$
C^{c} \cong\left(C \cap\left(X^{*} \backslash Y^{*}\right)\right) \cup\left(\mathbf{U}_{y \in C} \varphi(y)\right)
$$

namely,

$$
C^{c}=c l_{\mathscr{c}}\left(C \cap\left(X^{*} \backslash Y^{*}\right)\right)
$$

Proposition 1. ( $\left.X^{*}, \mathscr{T}\right)$ is minimal.

Proof. Let $\mathscr{W}$ be an open filter such that

$$
\cap\{U: U \in \mathscr{U}\}=\cap\left\{c l_{\sigma} U: U \in \mathscr{U}\right\}=\{x\}
$$

and let $V \in \mathscr{T}, x \in V$. We claim that there exists $U \in \mathscr{C}$ such that $V \supseteqq U$.

Case 1. Suppose $x=p^{*} \in Y^{*}$. Then there exists $\mathscr{W} \in \mathscr{K}$ with $\varphi(p) \subseteq \mathscr{W}$ such that $\mathscr{W} \cap(X \backslash Y) \subseteq V \cap(X \backslash Y)$ and such that if $y^{*} \in Y^{*}, \varphi(y) \subseteq \mathscr{Y}$ then $y^{*} \in V$. Then

$$
\mathscr{W} \supseteqq \cap\left(c l_{\sigma} U\right)^{c}=\varphi(p)
$$


so there exists $U \in \mathscr{U}$ such that $\mathscr{\mathscr { V }} \supseteqq\left(c l_{J} U\right)^{c}$. In particular,

$$
V \supseteqq \mathscr{V}^{-} \cap(X \backslash Y) \supseteqq U \cap\left(X^{*} \backslash Y^{*}\right) \text {. }
$$

However, if $z^{*} \in\left(U \cap Y^{*}\right) \backslash V$ then $\varphi(z) \nsubseteq \mathscr{V}$ but $\varphi(z) \subseteq\left(c l_{\sigma} U\right)^{c}$ since $U \in \mathscr{T}$. Thus if $z^{*} \in\left(U \cap Y^{*}\right) \backslash V$ then there exists $y \in\left(c l_{\sigma} U\right)^{c} \backslash \mathscr{Y}$, a contradiction. Thus $V \supseteqq\left(U \cap\left(X^{*} \backslash Y^{*}\right)\right) \cup\left(U \cap Y^{*}\right)=U$.

Case 2. Suppose $x \in X^{*} \backslash Y^{*}$. We may suppose that $V \cong X^{*} \backslash Y^{*}$ and in fact that $c l_{\varkappa} V \subseteq X^{*} \backslash Y^{*}$ since $Y$ is $\mathscr{K}$-closed in $X$. Then, again, there exists $U \in \mathscr{C}$ such that $V \cap\left(X^{*} \backslash Y^{*}\right) \supseteqq U \cap\left(X^{*} \backslash Y^{*}\right)$. Since $U \in \mathscr{T}$ and $c l_{\varkappa^{*}} V \subseteq X^{*} \backslash Y^{*}$ we must have that $U \subseteq X^{*} \backslash Y^{*}$ so that again $V \supseteqq U$.

Proposition 2. ( $\left.X^{*}, \mathscr{T}\right)$ is not functionally compact.

Proof. Let $A=\left\{y_{1}^{*}, y_{2}^{*}\right\}$. Suppose $\varphi\left(y_{1}\right)=\left\{x_{1}, x_{1}^{\prime}\right\}, \varphi\left(y_{2}\right)=\left\{x_{2}, x_{2}^{\prime}\right\}$. If $\mathscr{O}_{1}, \mathscr{O}_{1}^{\prime}, \mathscr{O}_{2}, \mathscr{O}_{2}^{\prime} \in \mathscr{K}$ are sets containing $x_{1}, x_{1}^{\prime}, x_{2}, x_{2}^{\prime}$, respectively then there are $U \in \mathscr{T}$ containing all $y^{*} \in Y^{*}$ with

$$
\varphi(y) \subseteq \mathscr{O}_{1} \cup \mathscr{O}_{1}^{\prime} \cup \mathscr{O}_{2} \cup \mathscr{O}_{2}^{\prime},
$$

and if $\mathscr{C}$ is the filter base of all such $U$ then $\cap U=\cap c l_{-} U=A$. However, there exist $\mathcal{O} \in \mathscr{T}, A \subseteq \mathcal{O}$ such that $\mathcal{O}$ does not contain any points $y^{*} \in Y^{*}$ unless $\varphi(y)$ is contained in a set of the form $\mathscr{O}_{1} \cup \mathscr{O}_{1}^{\prime}$ or one of the form $\mathscr{O}_{2} \cup \mathscr{O}_{2}^{\prime}$. Thus the filter generated by $\mathscr{U}$ does not contain all neighborhoods of $A$.

In [4] G. Viglino studies a property similar to functional compactness. $X$ is $C$-compact if for each closed set $A \subseteq X$ and each open cover $\mathscr{U}=\left\{U_{\alpha}\right\}$ of $A$ there exist $\alpha_{1}, \cdots, \alpha_{n}: c l_{X}\left(U_{\alpha_{1}} \cup \cdots \cup U_{\alpha_{n}}\right) \supseteqq A$. It is easy to see that if $X$ is $C$-compact then $X$ is functionally compact. We do not know whether the two properties are equivalent.

Urysohn's two examples to which we have referred can both be embedded as (nondense) subsets of functionally compact spaces. The one referred to in Example 1 can be embedded in $F$. If we let $Q$ be the quotient space obtained from two disjoint copies of $F$ by identifying the points $\alpha_{i}$ in the first with $\alpha_{i}$ in the second, $i=1,2, \cdots$, we get a functionally compact space in which Urysohn's minimal, noncompact space can be embedded, again as a nondense subset. This is the best that can be expected, since an absolutely closed space $C$ embedded in a Hausdorff space $H$ would have to be a closed subset of $H$, and hence not dense in $H$ is $H$ is functionally compact unless $C=H$. Also, since open and closed subsets of functionally compact 
spaces are functionally compact a nonfunctionally compact absolutely closed space can not be embedded as an open subset of a functionally compact set. We do not know whether for each Hausdorff $H$ there exists a functionally compact $X$ with $H \cong X$.

\section{BIBLIOGRAPHY}

1. Manuel P. Berri, Minimal topological spaces, Trans. Amer. Math. Soc. 108 (1963), 97-105.

2. Bourbaki, General topology, Addison-Wesley, 1966.

3. Chen-Tung Liu, Absolutely closed spaces, Trans. Amer. Math. Soc. 130 (1968), 86104.

4. Giovanni Viglino, C-compact spaces, (to appear in Duke Math J.)

Received February 21, 1969.

The University of Miami

Coral Gables, Florida 



\section{PACIFIC JOURNAL OF MATHEMATICS}

\section{EDITORS}

\author{
H. ROYDEN \\ Stanford University \\ Stanford, California \\ Richard Pierce \\ University of Washington \\ Seattle, Washington 98105
}

\author{
J. DugundJI \\ Department of Mathematics \\ University of Southern California \\ Los Angeles, California 90007 \\ BASIL GoRDON \\ University of California \\ Los Angeles, California 90024
}

\section{ASSOCIATE EDITORS}
E. F. BECKENBACH
B. H. Neumann
F. WOLF
K. YoSHIDA

\section{SUPPORTING INSTITUTIONS}

\author{
UNIVERSITY OF BRITISH COLUMBIA \\ CALIFORNIA INSTITUTE OF TECHNOLOGY \\ UNIVERSITY OF CALIFORNIA \\ MONTANA STATE UNIVERSITY \\ UNIVERSITY OF NEVADA \\ NEW MEXICO STATE UNIVERSITY \\ OREGON STATE UNIVERSITY \\ UNIVERSITY OF OREGON \\ OSAKA UNIVERSITY \\ UNIVERSITY OF SOUTHERN CALIFORNIA
}

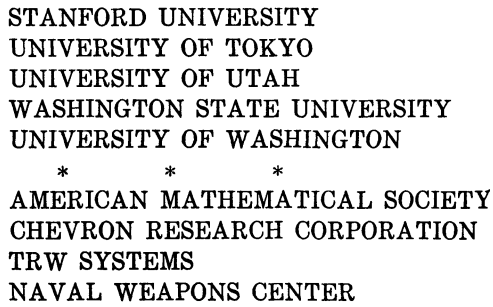

The Supporting Institutions listed above contribute to the cost of publication of this Journal, but they are not owners or publishers and have no responsibility for its content or policies.

Mathematical papers intended for publication in the Pacific Journal of Mathematics should be in typed form or offset-reproduced, double spaced with large margins. Underline Greek letters in red, German in green, and script in blue. The first paragraph or two must be capable of being used separately as a synopsis of the entire paper. It should not contain references to the bibliography. Manuscripts, in duplicate if possible, may be sent to any one of the four editors. Please classify according to the scheme of Math. Rev. 36, 1539-1546. All other communications to the editors should be addressed to the managing editor, Richard Arens, University of California, Los Angeles, California, 90024.

50 reprints are provided free for each article; additional copies may be obtained at cost in multiples of 50 .

The Pacific Journal of Mathematics is published monthly. Effective with Volume 16 the price per volume (3 numbers) is $\$ 8.00$; single issues, $\$ 3.00$. Special price for current issues to individual faculty members of supporting institutions and to individual members of the American Mathematical Society: $\$ 4.00$ per volume; single issues $\$ 1.50$. Back numbers are available.

Subscriptions, orders for back numbers, and changes of address should be sent to Pacific Journal of Mathematics, 103 Highland Boulevard, Berkeley, California, 94708.

PUBLISHED BY PACIFIC JOURNAL OF MATHEMATICS, A NON-PROFIT CORPORATION

Printed at Kokusai Bunken Insatsusha (International Academic Printing Co., Ltd.), 7-17, Fujimi 2-chome, Chiyoda-ku, Tokyo, Japan. 


\section{Pacific Journal of Mathematics}

\section{Vol. 31, No. $2 \quad$ December, 1969}

Efraim Pacillas Armendariz, Quasi-injective modules and stable torsion

classes..........................................

J. Adrian (John) Bondy, On Ulam's conjecture for separable graphs...

Vasily Cateforis and Francis Louis Sandomierski, On commutative rings over which the singular submodule is a direct summand for every module .....

Rafael Van Severen Chacon, Approximation of transformations with continuous

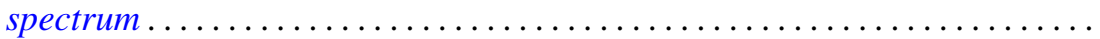

Raymond Frank Dickman and Alan Zame, Functionally compact spaces ...... 303

Ronald George Douglas and Walter Rudin, Approximation by inner

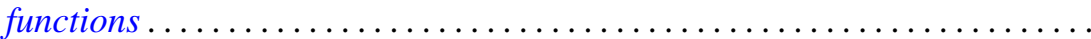

John Walter Duke, A note on the similarity of matrix and its conjugate

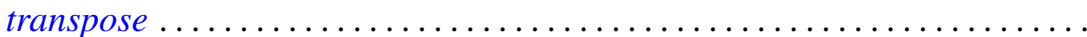

Micheal Neal Dyer and Allan John Sieradski, Coverings of mapping

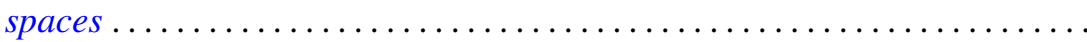

Donald Campbell Dykes, Weakly hypercentral subgroups of finite groups .....

Nancy Dykes, Mappings and realcompact spaces.....................

Edmund H. Feller and Richard Laham Gantos, Completely injective

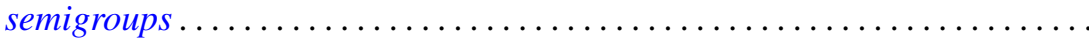

Irving Leonard Glicksberg, Semi-square-summable Fourier-Stieltjes

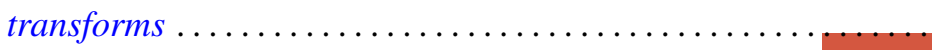

Samuel Irving Goldberg and Kentaro Yano, Integrability of almost cosymplectic structures...

Seymour Haber and Charles Freeman Osgood, On the sum $\sum\langle n \alpha\rangle^{-t}$ and numerical integration ..........................

Sav Roman Harasymiv, Dilations of rapidly decreasing functions ....

William Leonard Harkness and R. Shantaram, Convergence of a sequence of

transformations of distribution functions

Herbert Frederick Kreimer, Jr., A note on the outer Galois theory of rings ...

James Donald Kuelbs, Abstract Wiener spaces and applications to analysis. .

Roland Edwin Larson, Minimal $T_{0}$-spaces and minimal $T_{D}$-spaces...

A. Meir and Ambikeshwar Sharma, On Ilyeff's conjecture .

Isaac Namioka and Robert Ralph Phelps, Tensor products of compact convex sets....

James L. Rovnyak, On the theory of unbounded Toeplitz operators ....

Benjamin L. Schwartz, Infinite self-interchange graphs.......

George Szeto, On the Brauer splitting theorem...

Takayuki Tamura, Semigroups satisfying identity $x y=f(x$,

Kenneth Tolo, Factorizable semigroups .................. 